\title{
Relação Entre Mobilidade Diafragmática com Variáveis Clínicas e Funcionais em Pacientes com DPOC
}

\author{
Elizabeth do Canto Brancher, ${ }^{1}$ Eduardo Matias dos Santos Steidl, ${ }^{2}$ \\ Tamires Daros dos Santos, ${ }^{1}$ Maurício Tatsch Ximenes Carvalho, ${ }^{3}$ \\ Paulo Roberto Oliveira de Jesus, ${ }^{1}$ Adriane Schmidt Paqualoto, ${ }^{1}$ \\ Isabella Martins de Albuquerque, ${ }^{4}$ Renata Mancopes ${ }^{1}$
}

\section{RESUMO}

Avaliar se há relação entre a mobilidade diafragmática com o tempo de trânsito oral e faríngeo, pontuação do índice preditor de mortalidade Body mass index, airflow Obstruction, Dyspnea and Exercise capacity (Bode), dispneia e o estado de saúde em pacientes com doença pulmonar obstrutiva crônica (DPOC). Estudo transversal com avaliação dos seguintes desfechos: mobilidade diafragmática (ultrassonografia), tempo de trânsito oral e faríngeo (videofluoroscopia), preditor de mortalidade (pontuação no índice Bode), dispneia (escala do Medical Research Council modificada - mMRC) e estado de saúde (COPD Assessment Test). Participaram

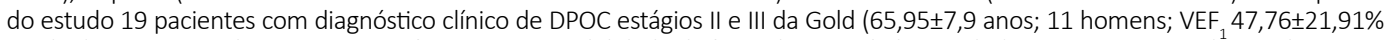
predito). Houve correlação negativa e forte entre a mobilidade diafragmática e o índice Bode ( $r=-0,822 ; p<0,0001)$, correlação negativa e moderada com o tempo de trânsito faríngeo na consistência líquida ( $r=-0,508 ; p=0,02)$ e correlação negativa e moderada com a dispneia $(r=-0,527 ; p=0,02)$. Na análise multivariada, a mobilidade diafragmática foi associada negativamente com o índice Bode $(\beta=-5,21 ; p<0,001 ;$ IC 95\%-7,28 a-3,15) e com o tempo de trânsito oral e faríngeo na consistência líquida $(\beta=-13,65 ; p<0,08$; IC 95\%-29,16 a 1,86). Não houve correlação entre a mobilidade diafragmática com o tempo de trânsito oral (consistência líquida e pastosa), com o tempo de trânsito faríngeo (consistência pastosa) e com o escore do CAT. Sugere-se que em pacientes com DPOC a mobilidade diafragmática está associada com o índice Bode, tempo de trânsito faríngeo na consistência líquida e dispneia.

Palavras-chave: Doença pulmonar obstrutiva crônica. Diafragma. Transtornos da deglutição. Ultrassonografia. Fluoroscopia.

\section{RELATIONSHIP BETWEEN DIAPHRAGMATIC MOBILITY WITH CLINICAL} AND FUNCTIONAL VARIABLES IN PATIENTS WITH COPD

\section{ABSTRACT}

To evaluate if there is a relationship between the diaphragmatic mobility with oral and pharyngeal transit time, predictor of mortality Body mass index, airflow Obstruction, Dyspnea and Exercise capacity (Bode) index score, dyspnea and health status in patients with Chronic Obstructive Pulmonary Disease (COPD). A cross-sectional study, with evaluation of following outcomes: diaphragmatic mobility (ultrasonography), oral and pharyngeal transit time (videofluroscopy), predictor of mortality (Bode index score), dyspnea (modified Medical Research Council - mMRC scale) and health status (COPD Assessment Test). Participated in this study 19 patients with clinical diagnosis of COPD stages Gold II or III (65.9 \pm 7.9 years of age, 11 male, FEV $47.76 \pm 21.91 \%$ predicted). There was a strong negative correlation between diaphragmatic mobility with BODE index score $(r=-0.822 ; p<0.0001)$, moderate negative correlation with pharyngeal transit time in the liquid consistency $(r=-0.508 ; p=0.02)$ and moderate negative correlation with dyspnea ( $r=-0.527 ; p=0.02)$. Multivariate regression analysis showed that the increase of diaphragmatic mobility was negatively associated with Bode index ( $\beta=-5.21 ; p<0.001 ; \mathrm{Cl} 95 \%-7.28$ to-3.15) and with pharyngeal transit time in the liquid consistency $(\beta=-13.65 ; p<0.08 ; C l 95 \%-29.16$ to 1.86$)$. There was no correlation between the diaphragmatic mobility and the oral transit time (liquid and pasty consistency), with the pharyngeal transit time (pasty consistency) and health status. It is suggested that in patients with COPD the diaphragmatic mobility is associated with Bode index, pharyngeal transit time in the liquid consistency and dyspnea. Keywords: Pulmonary disease. Chronic obstructive. Diaphragm. Deglutition disorders. Ultrasonography. Fluoroscopy.

RECEBIDO EM: 5/10/2019

MODIFICAÇÕES SOLICITADAS EM: 6/1/2021

ACEITO EM: 19/1/2021

\footnotetext{
${ }^{1}$ Universidade Federal de Santa Maria. Santa Maria/RS, Brasil.

2 Universidade Regional do Noroeste do Estado do Rio Grande do Sul - Unijuí. ljuí/RS, Brasil.

${ }^{3}$ Centro Universitário da Região da Campanha - Urcamp. Bagé/RS, Brasil.

${ }^{4}$ Autora correspondente. Universidade Federal de Santa Maria - Departamento de Fisioterapia e Reabilitação. Av. Roraima, 1000 - Bairro Camobi. Santa Maria/RS, Brasil. CEP 97105-900. http://lattes.cnpq.br/5579735303815692. http://orcid. org/0000-0002-7256-1881. albuisa@gmail.com
} 


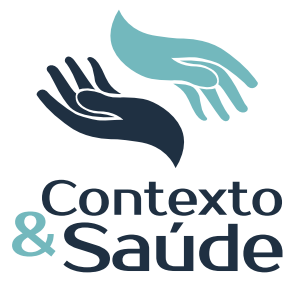

\section{INTRODUÇÃO}

A doença pulmonar obstrutiva crônica (DPOC) representa um importante problema de saúde pública na atualidade, pois compreende a quarta causa de mortalidade em âmbito mundial com a possibilidade de tornar-se a terceira até o ano de 2020 (GOLD, 2019). Entre os preditores envolvidos nessa estimativa, o reduzido estado de saúde de pacientes com DPOC contribui para o aumento no risco de mortalidade (Crisafulli et al., 2018).

A limitação ao fluxo aéreo é uma das principais características clínicas de pacientes com DPOC. Essa disfunção ventilatória ocorre em razão de um processo inflamatório pulmonar anormal, o qual é deflagrado pela exposição a partículas e gases nocivos (GOLD, 2019). Neste contexto, a avaliação espirométrica é utilizada para diagnosticar a DPOC e possibilitar a estratificação quanto à gravidade da obstrução do fluxo aéreo de acordo com a Global Initiative for Chronic Obstructive Lung Disease (Gold) nos seguintes estágios: Gold I ( $\geq 80 \%$ do valor predito do volume expiratório forçado no primeiro segundo $\left.-\mathrm{VEF}_{1}\right)$, II (50-79 \% do valor predito do $\mathrm{VEF}_{1}$ ), III (30-49 \% do valor predito do $\mathrm{VEF}_{1}$ ) e IV (<30\% do valor predito do $V E F_{1}$ ) (GOLD, 2019). Na evolução da doença, a degradação da elasticidade do parênquima pulmonar e a hiperinsuflação pulmonar são fatores limitantes da mobilidade diafragmática (MD) (SALITO et al., 2015). A modificação do deslocamento diafragmático, dificulta a ventilação, reduz a capacidade respiratória e ocasiona o aumento do principal sintoma da DPOC - a dispneia (PAULIN et al., 2007).

Em virtude das implicações clínicas da MD em pacientes com DPOC, estudos têm investigado sua possível associação com diferentes alterações causadas pela doença. Recentemente, Gonçalves et al. (2018) encontraram correlação negativa entre MD e o ângulo da curvatura torácica. Rocha et al. (2017) observaram que a $\mathrm{MD}$ apresentou correlação com a obstrução das vias aéreas, hiperinsuflação pulmonar, capacidade ventilatória e percepção de dispneia. Nesse contexto, o método no qual tem sido amplamente utilizado em razão de suas vantagens (segurança, viabilidade, repetibilidade e reprodutibilidade) para avaliação da MD em pacientes com DPOC, é a ultrassonografia do diafragma (BOUSSUGES et al., 2009).

Além das implicações musculares e respiratórias, outras importantes funções podem ser afetadas pela DPOC, como a deglutição (DE DEUS et al., 2014). Sabe-se que a respiração é fisiologicamente coordenada e relacionada com a deglutição, fator que permite um trânsito seguro de alimentos por meio da faringe, no entanto o desequilíbrio entre essas funções torna os pacientes com DPOC suscetíveis às modificações na dinâmica e coordenação da deglutição, resultando em aspiração, pneumonia, má nutrição, impacto no estado de saúde e óbito (LEE et al., 2017). Dessa forma, o desenvolvimento de complicações respiratórias pode estar relacionado com a alteração no tempo de trânsito oral e no tempo de trânsito faríngeo, que é avaliado por meio de um procedimento considerado padrão ouro: a videofluoroscopia (CASSIANI et al., 2015). Em contrapartida, a possível relação entre a MD e o tempo de trânsito oral, tempo de trânsito faríngeo, índice BODE (Body Mass-Index, Airflow Obstruction, Dyspnea and Exercise Capacity), dispneia e estado de saúde em pacientes com DPOC, ainda não foi investigada. 
O objetivo do presente estudo foi avaliar se há relação entre a MD com o tempo de trânsito oral, tempo de trânsito faríngeo, índice Bode, dispneia e o estado de saúde em pacientes com DPOC.

\section{METODOLOGIA}

Este é um estudo transversal conduzido na Unidade de Reabilitação do Hospital Universitário de Santa Maria (HUSM), Santa Maria, Rio Grande do Sul, Brasil, entre janeiro de 2016 e julho de 2017 e aprovado pelo Comitê de Ética em Pesquisa local sob CAAE $n^{\circ}$ 57250116.3.0000.5346. Todos os participantes foram informados com relação aos procedimentos e assinaram um Termo de Consentimento Livre e Esclarecido.

A amostra foi composta por pacientes com DPOC, recrutados durante a avaliação inicial conforme o seguinte critério: diagnóstico clínico de DPOC estágios II ou III, estabelecido de acordo com os critérios da Gold e clinicamente estáveis. Os critérios de exclusão definidos previamente compreenderam: pacientes com hemiparalisia ou paresia diafragmática, histórico de comprometimento neurológico, traqueostomizados, em uso de sonda para alimentação, com câncer de cabeça e/ou pescoço, tabagistas ativos, cardiopatas e/ou com hipertensão arterial não controlada, pacientes com doença hepática, obesos (IMC $\left.>30 \mathrm{~kg} / \mathrm{m}^{2}\right)$, histórico de cirurgia abdominal ou torácica, ou com distúrbio neuromuscular, musculoesquelético ou articular.

Todos os pacientes foram submetidos a dois dias de avaliação, com intervalo mínimo de 48 horas entre elas. No primeiro dia foi realizada a anamnese, exame físico, a aplicação do questionário COPD Assessment Test (CAT), escala Medical Research Council modificada (mMRC) e o teste de caminhada de seis minutos (TC6). No segundo dia foram feitas a avaliação da MD e a videofluoroscopia da deglutição.

\section{Espirometria}

A avaliação espirométrica foi realizada utilizando-se o espirômetro portátil MicroQuark ${ }^{\circledR}$ (Cosmed, Roma, Itália) no Ambulatório de Pneumologia do HUSM. O exame foi realizado de acordo com as diretrizes da American Thoracic Society/ European Respiratory Society (ATS; ERS, 2002) com determinação do volume expiratório forçado no primeiro segundo $\left(V_{E F}\right)$, capacidade vital forçada (CVF) e relação $V F_{1} / C V F$ após o uso de medicação broncodilatadora. Os valores obtidos foram comparados aos previstos para a população brasileira (PEREIRA, 2002).

\section{Questionário de estado de saúde na DPOC}

A versão em português do questionário CAT, validada para a população brasileira, foi aplicada sob a forma de entrevista para avaliar o estado de saúde (DA SILVA et al., 2013).

\section{Dispneia}

Para a avaliação da sensação de dispneia foi utilizada a versão validada para uso no Brasil da escala mMRC, cuja classificação varia de zero a 4, sendo 4 um valor indicativo de maior grau de dispneia (MOREIRA et al., 2009). 


\section{Teste de caminhada de seis minutos (TC6)}

O TC6 foi realizado de acordo com diretrizes internacionais por avaliadores treinados, em um corredor plano, coberto, medindo 30 metros de comprimento e 1,5 metros de largura, demarcados a cada 3 metros. O teste consistiu em uma caminhada, na qual o paciente era orientado a percorrer a maior distância possível (sem correr) por um período de seis minutos. Dois testes foram feitos no mesmo dia, com um intervalo de 30 minutos, visando a minimizar o efeito da aprendizagem, sendo o melhor desempenho utilizado para a análise (maior distância percorrida) (ATS; ERS, 2002).

\section{Mobilidade diafragmática (MD)}

A mensuração da MD foi realizada por intermédio do aparelho de ultrassom (Toshiba Medical Systems Corporation, SSA-590A, Tokyo, Japan) no modo B, sempre pelo mesmo médico radiologista no Serviço de Radiologia do HUSM. Durante o procedimento os pacientes estavam em decúbito dorsal, e na região subcostal direita um transdutor convexo de $3,5 \mathrm{MHz}$ foi posicionado com ângulo de incidência perpendicular ao eixo craniocaudal em direção à veia cava inferior. Inicialmente foi identificada a veia porta intra-hepática e, em seguida, o seu ramo esquerdo. A posição do ramo esquerdo foi marcada com o cursor do aparelho ao final de uma expiração e inspiração máximas, comandadas verbalmente pelo avaliador. O deslocamento craniocaudal desses pontos foi considerado a medida de mobilidade da hemicúpula diafragmática direita, sendo três medidas registradas para cada paciente e considerada para análise a de maior valor (GRAMS et al., 2014). Foram levados em conta os valores de referência para MD descritos por Boussuges et al. (2009).

\section{Tempo de trânsito oral e tempo de trânsito faríngeo}

A biomecânica da deglutição foi avaliada por meio das variáveis tempo de trânsito oral e tempo de trânsito faríngeo, que foram analisadas conforme protocolo proposto por Baijens et al. (2011). A avaliação ocorreu por intermédio da videofluoroscopia da deglutição, realizada por um médico radiologista e acompanhada por uma fonoaudióloga no Serviço de Radiologia do HUSM. Utilizou-se o equipamento Axiom Iconos R200 (Siemens Medical Systems, Forchheim, Alemanha) acoplado a um sistema computadorizado de gravação de imagem.

Os sujeitos foram avaliados na posição sentada, com projeção lateral (Figura 1C). Foi analisada a ingestão de alimentos contrastados com bário líquido (Barioge ${ }^{\circ}$ ) nas consistências líquida e pastosa. Na consistência líquida a água foi adicionada ao bário (15 $\mathrm{ml}$ de água para $15 \mathrm{ml}$ de bário), e na consistência pastosa a água foi adicionada ao bário e a um espessante ( $15 \mathrm{ml}$ de água, $15 \mathrm{ml}$ de bário e $5 \mathrm{ml}$ de espessante) (DROZDZ et al., 2012).

As variáveis em estudo foram analisadas por três avaliadores cegados, com experiência de cinco anos em análise de videofluoroscopia. 
Figura 1 - Ultrassonografia e videofluoroscopia

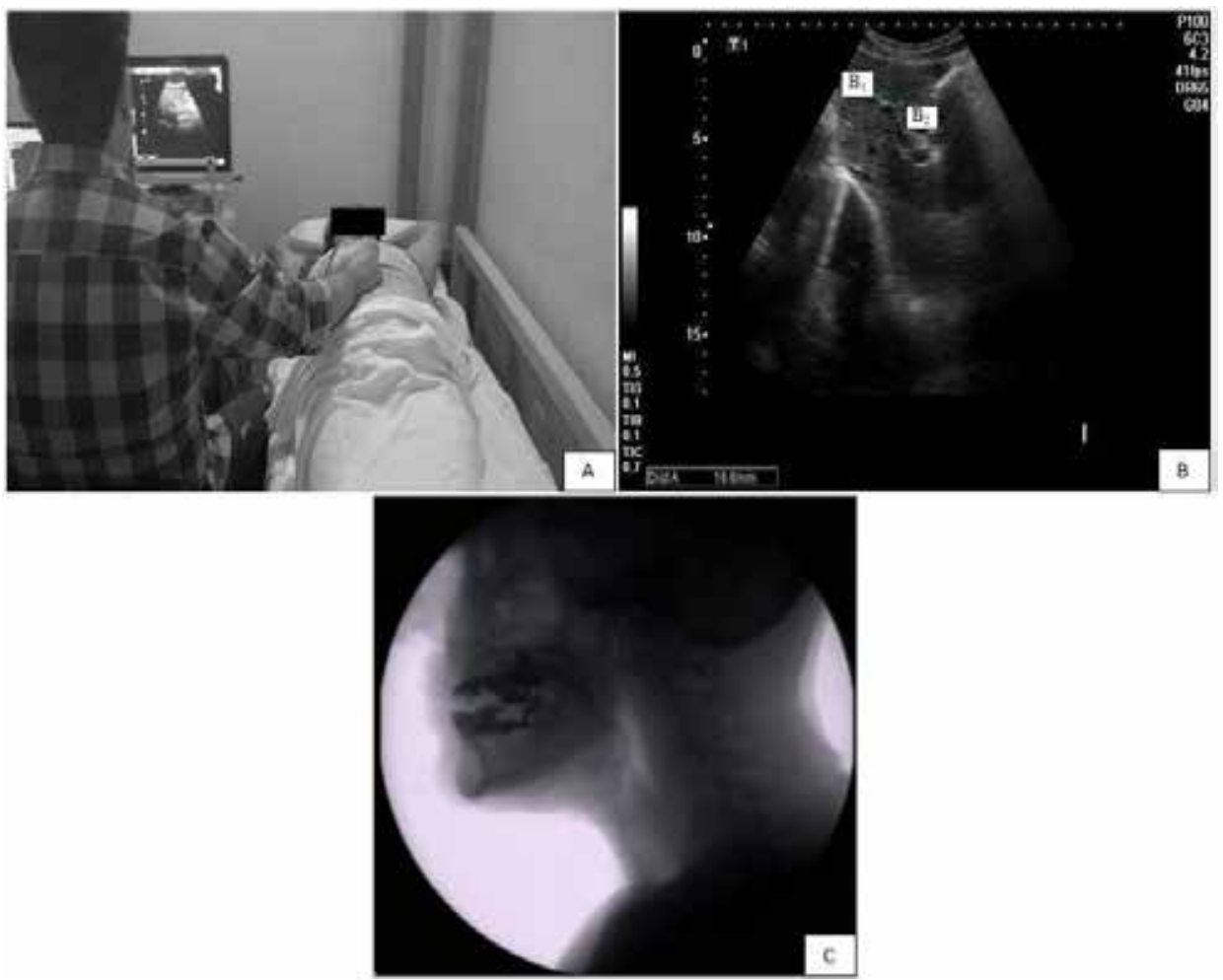

Em A, posicionamento do transdutor durante avaliação; em B, imagem ultrassonográfica, $B$ representa o cursor no momento da expiração máxima e $\mathrm{B}_{2}$ o cursor no momento da inspiração máxima; em C, imagem representativa do exame de videofluoroscopia em projeção lateral.

Fonte: Banco de dados dos autores.

\section{Índice BODE}

O risco de mortalidade foi avaliado pelo índice Bode. Para o cálculo do mesmo foram consideradas as variáveis: IMC, volume expiratório forçado no primeiro segundo, em porcentagem do previsto ( $V E F_{1} \%$ prev), pontuação na escala mMRC e distância percorrida no TC6. Os resultados obtidos pelos pacientes nas quatro variáveis foram pontuados e, após, divididos em quartis.

\section{Cálculo do tamanho da amostra}

Foi realizado pelo software BioEstat (versão 5.0), baseado em estudo piloto, quando foram incluídos os dez primeiros pacientes. Para detectar uma correlação de $r=-0,671$ entre as variáveis MD e a pontuação do índice preditor de mortalidade Bode, com um nível de significância de $5 \%(p<0,05)$, poder estatístico de $80 \%$ e assumindo possíveis perdas em $20 \%$, um tamanho amostral de 16 pacientes foi calculado.

\section{Análise estatística}

A análise estatística foi realizada utilizando-se o software Statistical Package for the Social Sciences, versão 20.0 (SPSS Inc., Chicago, IL, EUA). A normalidade das variáveis foi avaliada pelo teste de Shapiro-Wilk. As variáveis contínuas foram apresentadas em média e desvio padrão e as categóricas em frequências absolu- 
tas e porcentagens. A concordância entre os avaliadores das variáveis da videofluoroscopia foi testada mediante a média obtida para cada variável pelo teste de Wilcoxon. A correlação entre a MD com as demais variáveis foi verificada pelo coeficiente de correlação de Pearson, sendo consideradas correlações fracas (valores de r entre 0,10 e 0,39), moderadas (valores de r entre 0,40 e 0,69) e fortes (valores de $r$ entre 0,70 e 1,00). A análise de regressão linear múltipla foi realizada para determinar se a MD estava associada com as demais variáveis do estudo. Primeiramente fez-se uma pré-seleção das variáveis por meio de uma análise de regressão linear simples, sendo o nível de significância adotado $p \leq 0,25$. Após, as variáveis com significância estatística foram inseridas no modelo e a análise de regressão linear múltipla realizada, considerando-se $p \leq 0,10$.

\section{RESULTADOS}

Foram incluídos neste estudo 19 pacientes com DPOC (11 homens) com obstrução moderada a grave das vias aéreas ( $V E F_{1} \% 47,76 \pm 21,91$ do previsto). Não ocorreram exclusões após o início das avaliações. A Tabela 1 apresenta a caracterização geral da amostra.

Tabela 1 - Características gerais dos pacientes

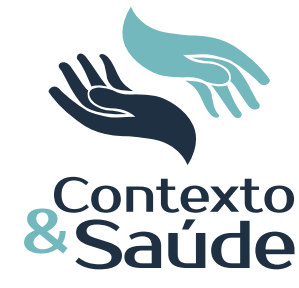

\begin{tabular}{|c|c|}
\hline Características & $\mathrm{n}=19$ \\
\hline Sexo, M/F & $11 / 8$ \\
\hline Idade (anos) & $65,95 \pm 7,9$ \\
\hline $\mathrm{IMC}\left(\mathrm{kg} / \mathrm{m}^{2}\right)$ & $24,62 \pm 4,85$ \\
\hline $\mathrm{IMM}, \mathrm{kg} / \mathrm{cm}^{2}$ & $18,13 \pm 2,25$ \\
\hline GOLD (II/III) & $7 / 12$ \\
\hline $\operatorname{VEF}_{1}$ (\% predito) & $47,76 \pm 21,91$ \\
\hline DPTC6 (m) & $326,72 \pm 88,72$ \\
\hline Escore (mMRC) & $2,16 \pm 0,69$ \\
\hline CAT & $17,58 \pm 8,08$ \\
\hline Índice Bode & $3,95 \pm 1,90$ \\
\hline \multicolumn{2}{|l|}{ Quartil } \\
\hline Quartil 1, n (\%) & $4(21,0)$ \\
\hline Quartil 2, n (\%) & $8(42,1)$ \\
\hline Quartil 3, n (\%) & $6(31,6)$ \\
\hline Quartil 4, n (\%) & $1(5,3)$ \\
\hline Mobilidade diafragmática (mm) & $37,84 \pm 13,87$ \\
\hline \multicolumn{2}{|l|}{ Consistência líquida } \\
\hline Tempo de trânsito oral (s) & $1,14 \pm 1,2$ \\
\hline Tempo de trânsito faríngeo (s) & $0,84 \pm 0,25$ \\
\hline \multicolumn{2}{|l|}{ Consistência pastosa } \\
\hline Tempo de trânsito oral (s) & $1,06 \pm 0,54$ \\
\hline Tempo de trânsito faríngeo (s) & $0,83 \pm 0,25$ \\
\hline
\end{tabular}

IMC: índice de massa corpórea; IMM: índice de massa magra; Gold: Global Initiative for Chronic Obstructive Lung Disease; DPTC6: distância percorrida no teste de caminhada de 6 minutos; mMRC: escala modified Medical Research Council; CAT: COPD Assessment Test; Índice Bode: Body mass index, Obstruction, Dyspnea and Exercise capacity.

Fonte: Banco de dados dos autores. 
A MD apresentou correlação negativa e forte com o índice Bode ( $r=-0,822$; $p<0,0001$ ) (Figura 2), correlação negativa e moderada com a dispneia ( $r=-0,527$; $p=0,02$ ) (Figura 3) e com o tempo de trânsito faríngeo na consistência líquida $(r=-0,508 ; p=0,02)$ (Figura 4). Não foram encontradas correlações entre a MD com o tempo de trânsito oral na consistência líquida e pastosa, respectivamente $(r=0,208 ; p=0,409$ e $r=-0,323 ; p=0,177)$, com o tempo de trânsito faríngeo na consistência pastosa $(r=-0,232 ; p=0,339)$ e com o escore do CAT $(r=-0,367$; $p=0,122$ ).

Nas análises entre os avaliadores da videofluoroscopia observou-se que para cada variável considerada os avaliadores obtiveram 100\% de concordância (Kappa=1,00), o que indica concordância quase perfeita.

Figura 2 - Correlação entre mobilidade diafragmática e o índice Bode

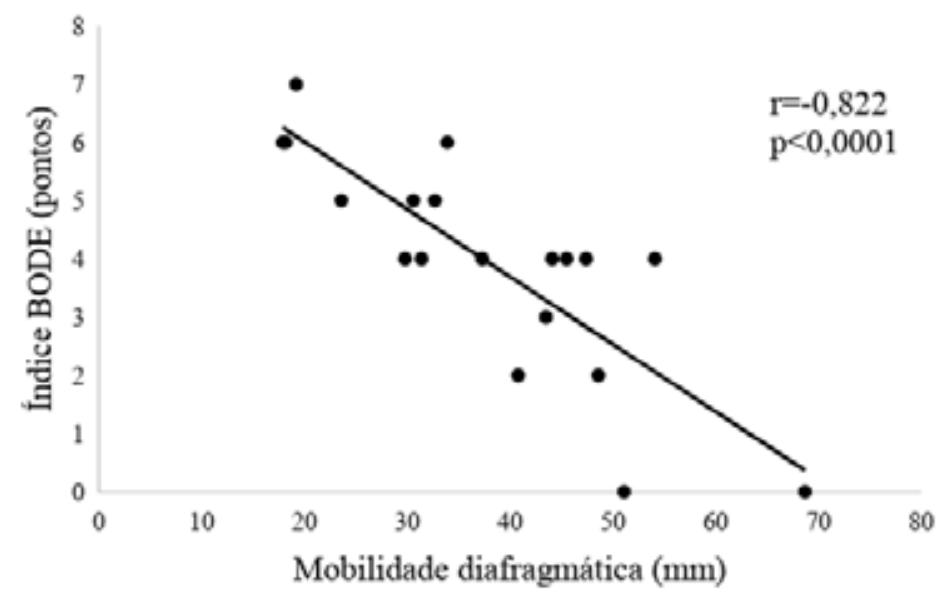

Fonte: Banco de dados dos autores.

Figura 3 - Correlação entre mobilidade diafragmática e dispneia

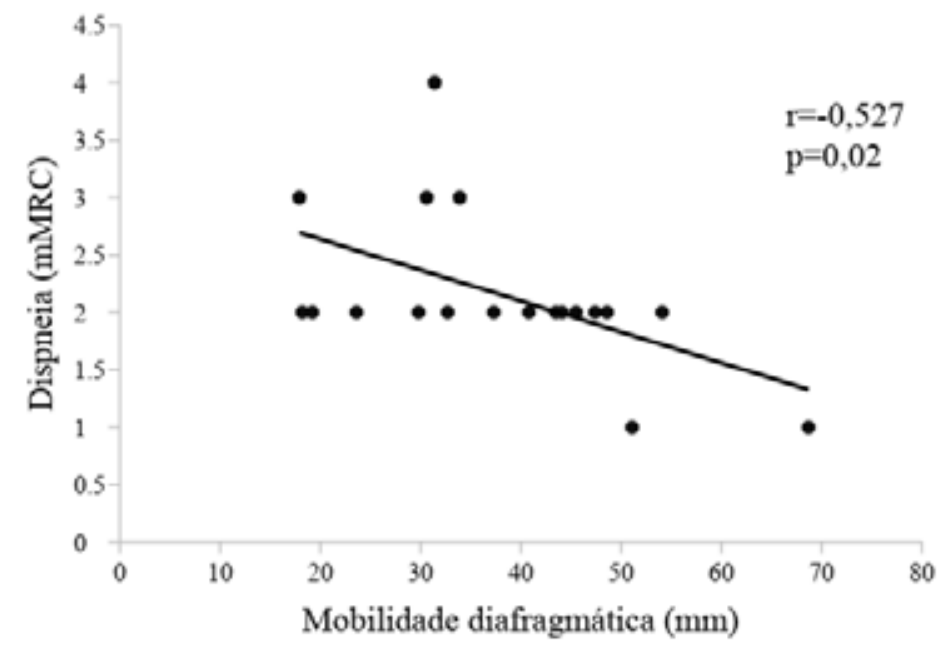

Fonte: Banco de dados dos autores. 
Figura 4 - Correlação entre mobilidade diafragmática e tempo de trânsito faríngeo na consistência líquida

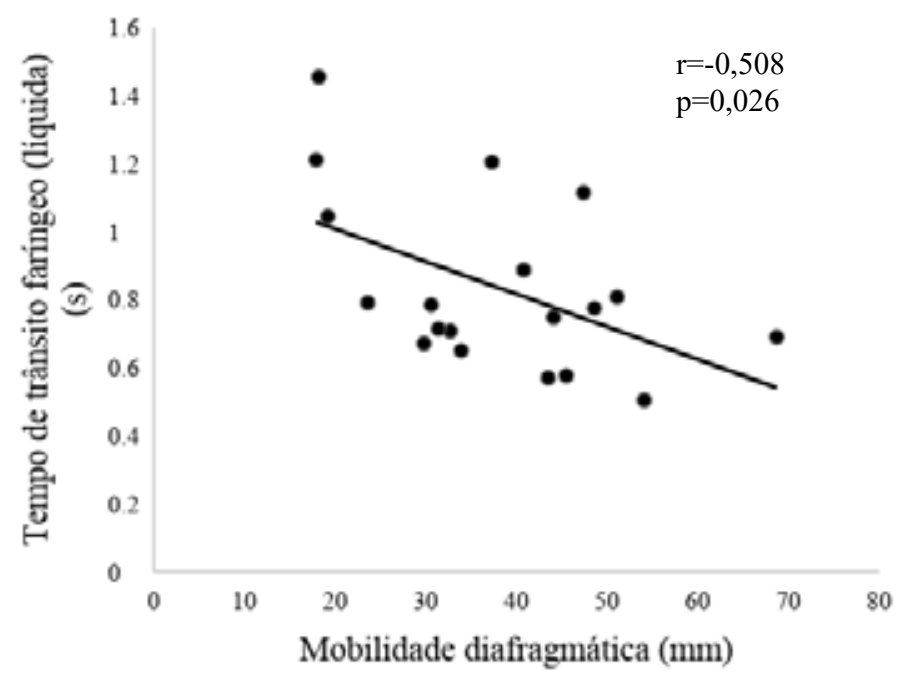

Fonte: Banco de dados dos autores.

$\mathrm{Na}$ análise multivariada, a MD foi associada negativamente com o índice Bode ( $\beta=-5,21 ; p<0,001 ;$ IC $95 \%-7,28$ a-3,15) e com o tempo de trânsito faríngeo na consistência líquida ( $\beta=-13,65 ; p<0,08 ;$ IC $95 \%-29,16$ a 1,86), explicando $70 \%$ da variância.

\section{DISCUSSÃO}

O presente estudo demonstrou que a MD apresentou uma correlação negativa e forte com o índice Bode, correlação negativa e moderada com a dispneia, assim como com o tempo de trânsito faríngeo na consistência líquida em pacientes com DPOC. Foi observado que a MD foi preditora de mortalidade e do aumento do tempo de trânsito faríngeo na consistência líquida. Por outro lado, a MD não apresentou relação com o tempo de trânsito oral nas consistências líquida e pastosa, com o tempo de trânsito faríngeo na consistência pastosa e com o escore do CAT. Ademais, observou-se redução da MD em relação aos valores preditos.

A avaliação da MD em pacientes com DPOC é relevante clinicamente, pois sua redução tem sido associada ao aumento da dispneia (GONÇALVES et al., 2018; ROCHA et al., 2017), intolerância ao exercício (PAULIN et al., 2007) e crescimento do ângulo da curvatura torácica (GONÇALVES et al., 2018), sendo, assim, de grande importância para a assistência do fisioterapeuta. Apesar da escassez de evidências, que investigam a relação entre MD e o índice Bode, destaca-se o estudo de Yamaguti et al. (2009), cujos achados sugerem que pacientes com DPOC e disfunção diafragmática apresentam maior risco de mortalidade. Semelhantemente ao estudo anterior, nossos achados sinalizaram que quanto menor a MD maior é a pontuação no índice Bode. Além da correlação observada, destaca-se que o índice Bode é preditor da MD. A aplicabilidade clínica 
desses achados reside no fato de o índice Bode ser um preditor de mortalidade em pacientes com DPOC (PRUDENTE et al., 2018), fortalecendo a sua utilização na prática clínica.

Ainda, no presente estudo, foi encontrada uma correlação negativa e moderada da MD com a dispneia. Em decorrência de modificações no posicionamento diafragmático, ocorre um comprometimento da ventilação e, consequentemente, um aumento da dispneia (ALTER et al., 2017). Em conformidade com os nossos achados, Rocha et al. (2017) também encontraram a mesma correlação, no entanto é importante mencionar que os referidos autores avaliaram a MD por meio da radiografia de tórax, enquanto, em nosso estudo, a mensuração foi conduzida por meio da ultrassonografia do diafragma.

Durante a deglutição em sujeitos saudáveis, ocorre uma apneia preventiva e, após, a respiração é retomada com uma expiração (MACHADO et al., 2015). Dessa maneira, alterações nos padrões respiratórios e ventilatórios podem apresentar implicações na coordenação entre deglutição e respiração afetando diretamente pacientes com DPOC (DE DEUS, 2014) e, consequentemente, apresentando implicações diretas para a conduta do fisioterapeuta, pois é um importante fator de risco para exacerbações da doença (NAGAMI et al., 2017). Outro estudo demonstrou que pacientes com DPOC apresentam um prolongamento do tempo de trânsito faríngeo na ingestão de consistências líquida e pastosa (DE DEUS et al., 2014). Semelhantemente, Tomasi (2018) analisou a fase oral de 25 indivíduos com DPOC e observou um tempo de trânsito oral aumentado, tanto na consistência liquida (2,09 segundos) quanto na pastosa (1,61 segundos).

De acordo com estudo de revisão crítica, há uma divergência quanto à definição do tempo de trânsito oral em indivíduos saudáveis. Os tempos encontrados na literatura variam de 0,35 segundos a 1,54 segundos para líquido e de 0,39 segundos a 1,05 segundos para a consistência pastosa (SOARES et al., 2015). Em relação ao tempo de trânsito faríngeo na consistência líquida e pastosa, Vale-Prodomo (2010) considera o valor de normalidade de 0,71 segundos. Assim, quando nossos achados são comparados aos descritos na literatura, observamos que o tempo de trânsito oral na consistência líquida foi classificado como normal (1,14 segundos), porém para a consistência pastosa foi considerado um pouco acima do limite de normalidade estabelecido (1,06 segundos). Ademais, observou-se um aumento do tempo de trânsito faríngeo na consistência líquida $(0,84$ segundos) e na consistência pastosa ( 0,83 segundos).

Até o limite do nosso conhecimento, este é o primeiro estudo a encontrar relação entre MD com o tempo de trânsito faríngeo em pacientes com DPOC. Nossos achados demonstraram que quanto menor a MD maior é o tempo de trânsito faríngeo na consistência líquida. Além dessa associação, foi constatado que a MD é preditora do aumento do tempo de trânsito faríngeo na consistência líquida. Prováveis explicações para os referidos achados residem na proximidade existente entre as funções centrais da deglutição e a respiração; desse modo, infere-se que a duração do tempo de trânsito faríngeo no paciente com DPOC pode estar associada à geração de uma pressão glótica insuficiente, considerando a reduzida função pulmonar desses pacientes (CASSIANI et al., 2015). O estímulo aferente do sistema respiratório modifica a resposta eferente da de- 
glutição (GROSS et al., 2009), embora tal mecanismo não tenha sido avaliado no presente estudo.

Outro achado do nosso estudo refere-se à ausência de correlação entre a $\mathrm{MD}$ e o tempo de trânsito faríngeo na consistência pastosa. Este resultado, conforme sugerido pela literatura, pode estar relacionado à viscosidade e ao volume dos alimentos utilizados para avaliação, que podem ter impacto nos aspectos sensoriais e motores da deglutição e, assim, afetar os parâmetros de duração (COSTA, 2018). Hipotetiza-se que outro possível fator pode ser a relação fisiológica existente entre o tempo de trânsito faríngeo e oral na consistência pastosa. Assim como não foi observada uma relação entre a MD e o tempo de trânsito faríngeo na consistência pastosa, não houve com o tempo de trânsito oral na mesma consistência (CASSIANI et al., 2011).

No presente estudo, a MD não apresentou correlação com o tempo de trânsito oral nas consistências avaliadas. Esses resultados corroboram o estudo de Cassiani et al. (2015), que também não observaram alterações no tempo de trânsito oral durante a deglutição em pacientes com DPOC. Esse achado pode ser atribuído ao fato de que a fase oral da deglutição é controlada voluntariamente, diferente do controle da fase faríngea, o qual ocorre em resposta à estimulação da faringe pelo bolo alimentar (LANG et al., 2014).

Nosso estudo não encontrou correlação da MD e a pontuação do questionário CAT, ou seja, a MD parece não estar associada com o impacto da doença no estado de saúde desses pacientes. É importante mencionar que em nossa amostra a média da pontuação no questionário CAT foi de 17,58, ou seja, a maioria dos pacientes apresentou um moderado impacto da doença no estado de saúde. Além disso, o referido instrumento destaca-se por ser amplamente utilizado na prática clínica do fisioterapeuta, em razão de ser curto, simples e de fácil compreensão pelo paciente (KARLOH et al., 2016), assim como ser preditor de exacerbação em pacientes com DPOC (GULART et al., 2017).

Nosso estudo apresenta algumas limitações. Primeiramente, por tratar-se de um estudo de delineamento transversal, os achados referentes à relação entre menor MD com os demais desfechos é restrito ao momento avaliado. Em segundo lugar, a amostra foi composta por pacientes nos estágios II e III; assim, nossos achados não podem ser extrapolados para todos os graus de comprometimento da DPOC. Em terceiro lugar, não foi realizada a avaliação dos volumes pulmonares estáticos pela pletismografia, fator que inviabilizou a investigação da correlação com essas variáveis.

\section{CONCLUSÃO}

O presente estudo demonstrou que a MD está associada ao índice Bode, à dispneia e ao tempo de trânsito faríngeo na consistência líquida em pacientes com DPOC. Em contrapartida, a MD não apresentou relação com o tempo de trânsito oral nas duas consistências avaliadas, com o tempo de trânsito faríngeo na consistência pastosa e com o escore do CAT. 


\section{AGRADECIMENTO}

Agradecemos à Coordenação de Aperfeiçoamento de Pessoal de Nível Superior (Capes) pelo apoio financeiro.

\section{REFERÊNCIAS}

ALTER, A. et al. Neuromuscular weakness in chronic obstructive pulmonary disease: chest wall, diaphragm, and peripheral muscle contributions. Current Opinion in Pulmonary Medicine, v. 23, n. 2, p. 129-138, 2017.

ATS; ERS. American Thoracic Society; European Respiratory Society. ATS/ERS Statement on respiratory muscle testing. American Journal of Respiratory and Critical Care Medicine, v. 166, n. 4, p. 518-624, 2002.

Baijens, L.W. et al. Swallowing in parkinson patients versus healthy controls: reliability of measurements in videofluoroscopy. Gastroenterology Research and Practice, v. 2011, 2011. doi: https://doi.org/10.1155/2011/380682.

BOUSSUGES, A. et al. Diaphragmatic motion studied by m-mode ultrasonography: methods, reproducibility, and normal values. Chest, v. 135, n. 2, p. 391-400, 2009.

CASSIANI, R. A. et al. Oral and pharyngeal bolus transit in patients with chronic obstructive pulmonary disease. International Journal of Chronic Obstructive Pulmonary Disease, v. 10, p. 489-96, 2015.

CASSIANI, R. A. et al. The relationship between the oral and pharyngeal phases of swallowing. Clinics, v. 66, n. 8, p. 1.385-1.388, 2011.

COSTA, M. M. B. Neural control of swallowing. Arquivos de Gastroenterologia, v. 55, Suppl 1, p. 61-75, 2018.

Crisafulli, E. et al. Clinical variables predicting the risk of a hospital stay for longer than 7 days in patients with severe acute exacerbations of chronic obstructive pulmonary disease: a prospective study. Respiratory Research, v. 19, n. 1, p. 261, 2018.

DA SILVA, G. P. et al. Portuguese-language version of the COPD Assessment Test: validation for use in Brazil. Jornal Brasileiro de Pneumologia, v. 39, n. 4, p. 402-8, 2013.

DE DEUS, C. R. et al. Swallowing transit times and valleculae residue in stable chronic obstructive pulmonary disease. BMC Pulmonary Medicine, v. 14, n. 62, p. 1-12, 2014.

DROZDZ, D. R. C. et al. Pharyngeal swallowing phase and chronic cough. International Archives of Otorhinolaryngology, v. 16, n. 4, p. 502-8, 2012.

GOLD. Global Initiative for Chronic Obstructive Lung Disease. GOLD 2019 Global Strategy for the Diagnosis, Management and Prevention of COPD. 2019. Disponível em: https:// goldcopd.org/wp-content/uploads/2018/11/GOLD-2019-POCKET-GUIDE-FINAL_WMS. pdf. Acesso em: 21 mar. 2019.

GONÇALVES, M. A. et al. Comparison of diaphragmatic mobility between COPD patients with and without thoracic hyperkyphosis: a cross-sectional study. Jornal Brasileiro de Pneumologia, v. 44, n. 1, p. 5-11, 2018.

GRAMS, S. T. et al. Assessment of the reproducibility of the indirect ultrasound method of measuring diaphragm mobility. Clinical Physiology and Functional Imaging, v. 34, n. 1, p. 18-25, 2014.

GROSS, R. D. et al. The coordination of breathing and swallowing in chronic obstructive pulmonary disease. American Journal of Respiratory and Critical Care Medicine, v. 179, n. 7, p. 559-656, 2009.

GULART, A. A. et al. Does the COPD assessment test reflect functional status in patients with COPD? Chronic Respiratory Disease, v. 14, n. 1, p. 37-44, 2017.

$\mathrm{KARLOH}, \mathrm{M}$. et al. The COPD assessment test: what do we know so far?: a systematic review and meta-analysis about clinical outcomes prediction and classification of patients into GOLD stages. Chest, v. 149, n. 2, p. 413-425, 2016. 
Lang, I. M. et al. Role of peripheral reflexes in the initiation of the esophageal phase of swallowing. American Journal Physiology-Gastrointestinal and Liver Physiology, v. 306, n. 8, p. 728-37, 2014.

LEE, H. et al. Different impacts of respiratory symptoms and comorbidities on COPD-specific health-related quality of life by COPD severity. International Journal of Chronic Obstructive Pulmonary Disease, v. 12, p. 3.301-3.310, 2017.

MACHADO, J. R. S. et al. Efeitos do exercício muscular respiratório na biomecânica da deglutição de indivíduos normais. Revista Cefac, v. 17, n. 6, p. 1.909-1.915, 2015.

MOREIRA, G. L. et al. Portuguese-language version of the Chronic Respiratory Questionnaire: a validity and reproducibility study. Jornal Brasileiro de Pneumologia, v. 35, n. 8, p. 737-44, 2009.

NAGAMI, S. et al. Breathing - swallowing discoordination is associated with frequent exacerbations of COPD. BMJ Open Respiratory Research, v. 4, n. 1, p. 1-9, 2017.

PAULIN, E. et al. Influence of diaphragmatic mobility on exercise tolerance and dyspnea in patients with COPD. Respiratory Medicine. v. 101, n. 10, p. 2.113-2.118, 2007.

PEREIRA, C. A. Spirometry. Jornal Brasileiro de Pneumologia, v. 28, n. 1, p. 1-82, 2002.

PRUDENTE, R. et al. Predictors of mortality in patients with COPD after 9 years. International Journal of COPD, v. 13, n. 1, p. 3.389-3.398, 2018.

ROCHA, F. R. et al. Diaphragmatic mobility: relationship with lung function, respiratory muscle strength, dyspnea, and physical activity in daily life in patients with COPD. Jornal Brasileiro de Pneumologia, v .43, n. 1, p. 32-7, 2017.

SALITO, C. et al. Alterations of diaphragm and rib cage morphometry in severe COPD patients by CT analysis. Conf Proc The IEEE Engineering in Medicine and Biology Society, p. 6.390-6.393, 2015.

SOARES, T. J. et al. Tempo de trânsito oral: revisão crítica da literatura. ABCD. Arquivos Brasileiros de Cirurgia Digestiva, São Paulo, v. 28, n. 2, p. 144-147, 2015.

TOMASI, L. L. Análise da fase oral da deglutição em indivíduos com doença pulmonar obstrutiva crônica. 2018. Dissertação (Mestrado) - Universidade Federal de Santa Maria, Santa Maria, 2018.

VALE-PRODOMO, L. P. Caracterização videofluoroscópica da fase faríngea da deglutição. 2010. Tese (Doutorado) - Fundação Antônio Prudente, São Paulo, 2010.

YAMAGUTI, W. P. S. et al. Diaphragmatic dysfunction and mortality in patients with COPD. Jornal Brasileiro de Pneumologia, v. 35, n. 12, p. 1.174-1.181, 2009. 